\title{
Propellant Budget Calculation of Geostationary Satellites
}

\author{
${ }^{1}$ Şenol Gülgönül and *1,2,3,4, Nedim Sözbir \\ ${ }^{1}$ BMC Power Engineering Department, İstanbul, Turkey \\ ${ }^{*}$ Faculty of Engineering, Department of Mechanical Engineering Sakarya University, Turkey \\ ${ }^{3}$ Türksat A.Ş. Gölbaşı, Ankara, Turkey \\ ${ }^{4}$ Tübitak MAM Gebze, Kocaeli, Turkey
}

\begin{abstract}
Propellant budget of the geostationary satellites has to be calculated during preliminary design phase to properly size propellant tanks, mass and dimensions of the satellite. Lifetime of the satellite depends on the propellant budget. A guideline for calculation of propellant budget of geostationary satellites is presented. Proposed method has enough accuracy for initial design phase of the satellite.
\end{abstract}

Keywords: Propellant budget; Satellite; Geostationary

\section{Introduction}

A Geostationary satellite is launched into space on a rocket, and once there it is inserted into the geostationary orbit and is maintained in that orbit by means of thrusters onboard the satellite itself. Satellites launched in the late 1980s and 1990s typically have an integrated propulsion system that use a bipropellant combination of monomethyl hydrazine as fuel and nitrogen tetroxide as oxidizer. The specific impulse is about 300 seconds and fuel margin not used for the apogee maneuver can be applied to station keeping [1-8].

Propellant budget calculation is an important analysis for sizing of geostationary satellite in preliminary design phase (PDR). Propellant budget defines the lifetime, mass of the satellite, propellant tank volumes and dimensions. In this study, a guideline is presented for the calculation of the propellant budget of geostationary satellite. Proposed approach has acceptable accuracy based on experiences and can be used for the preliminary design phase.

\section{Calculation Method}

The law of physics on which rocket propulsion is based is called the principle of momentum. According to this principle, the time rate of change of the total momentum of a system of particles is equal to the net external force. The momentum is defined as the product of mass and velocity. If the net external force is zero, then the principle of momentum becomes the principle of conservation of momentum and the total momentum of the system is constant. To balance the momentum conveyed by the exhaust, the rocket must generate a momentum of equal magnitude

*Corresponding author: Address: Faculty of Engineering, Department of Mechanical Engineering Sakarya University, 54187, Sakarya TURKEY. E-mail address: sozbir@sakarya.edu.tr, Phone: +902642955866 
but in the opposite direction and thus it accelerates forward. The satellite has its own propulsion system that is used for orbit insertion, station keeping, momentum wheel desaturation, and attitude control. The propellant required to perform a maneuver with a specified velocity increment $\Delta \mathrm{M}$ is given by the "rocket equation" [1,2]

$$
\Delta M=M\left[1-e^{-\Delta V /\left(g \cdot I_{s p} \cdot \eta\right)}\right]
$$

where

$\Delta \mathrm{V} \quad$ : velocity change,

$\mathrm{M} \quad$ : mass of the satellite before the maneuver,

$\Delta \mathrm{M} \quad$ : change in mass due to velocity increment,

Isp : Specific impulse of the thruster,

$\eta \quad$ : efficiency of the maneuver,

g : gravitational constant $=9.80665 \mathrm{~m} / \mathrm{s}^{2}$

In the case of a geostationary satellite, the satellite must perform a critical maneuver at the apogee of the transfer orbit at the synchronous altitude of $35,786 \mathrm{~km}$ to simultaneously remove the inclination and circularize the orbit. The transfer orbit has a perigee altitude of about $200 \mathrm{~km}$ and an inclination roughly equal to the latitude of the launch site. To minimize the required velocity increment, it is thus advantageous to have the launch site as close to the equator as possible [1-9].

Required velocity increments for orbit raising, East-West, North-South, relocation and de-orbiting maneuvers have to be calculated as a part of the mission analysis. Manufacturers provide specific impulses of thrusters. For example, specific impulse of Airbus Safran Launchers GmbH 10N thruster is 292 second and $400 \mathrm{~N}$ apogee motor has 318 second nominal specific impulse.

Velocity change required for orbit raising depends on the launcher selection. Launcher leaves the satellite at Geostationary Transfer Orbit (GTO) and satellite performs several apogee maneuvers (typically three) to go Geostationary Orbit (GEO). Space X Falcon-9 and Ariane-5 launcher's GTO orbit parameters are presented at Table.1 [10,11].

Table 1. Launcher Performances

\begin{tabular}{|l|c|c|c|}
\hline Launcher & Inclination (degree) & Perigee $(\mathrm{km})$ & Apogee $(\mathrm{km})$ \\
\hline Falcon-9 & 28.5 & 185 & 35,786 \\
\hline Ariane-5 & 6 & 250 & 35,943 \\
\hline
\end{tabular}

Velocity of the satellite on elliptic orbit is given by 
$V=\sqrt{G M\left(\frac{2}{R}-\frac{1}{a}\right)}$

where

GM : Earth Gravitational Constant, $398600.4418 \mathrm{~km}^{3} / \mathrm{s}^{2}$

$\mathrm{R}$ : Distance of the satellite to the center of the Earth, $\mathrm{km}$

a : Semi-major axis of the satellite orbit, $\mathrm{km}$

Apogee maneuvers circularize the elliptical GTO orbit to GEO orbit and correct the inclination between these two orbit planes (Fig. 1). These maneuvers are performed at apogee of the GTO orbit to have minimal velocity change [1-9].

Semi-major of the GEO orbit is $42164 \mathrm{~km}$ and the velocity at GEO orbit can be calculated using (2)

$V_{s}=\sqrt{\frac{G M}{42164}}=3074.7 \mathrm{~m} / \mathrm{s}$

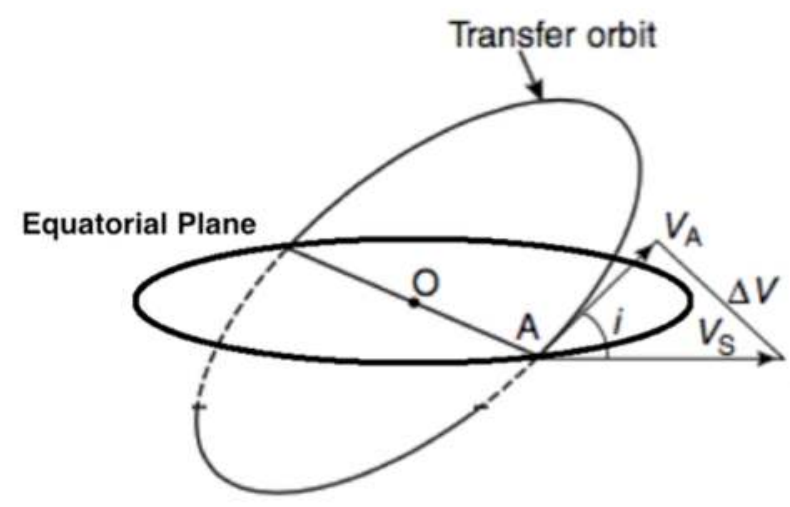

Figure 1. GTO to GEO orbit maneuver

The radius of the Earth can be assumed as $6378 \mathrm{~km}$. For example, semi-major axis of the GTO orbit for Ariane- 5 can be calculated by using altitude of apogee and perigee.

$a=\frac{250+35943}{2}+6378=24474.5 \mathrm{~km}$

Apogee velocity of the satellite is calculated using (2). 
$V_{A}=\sqrt{G M\left(\frac{2}{35943+6378}-\frac{1}{244745}\right)}=1597.1 \mathrm{~m} / \mathrm{s}$

Finally, the required $\Delta \mathrm{V}$ increment for Ariane- 5 can be calculated using cosine rule.

$$
\Delta V=\sqrt{V_{a}^{2}+V_{S}^{2}-2 V_{a} V_{S} \cos (i)}=1495.7 \mathrm{~m} / \mathrm{s}
$$

Velocity increment for East-West maneuvers depends on longitude of the satellite. Due to gravity distribution of the Earth 75 East and 105 West are stable orbital locations for geostationary satellite (see Fig. 2). Longitude acceleration for 42 degree East is given as $0.00171 \mathrm{deg} / \mathrm{day}^{2}$. Yearly required $\Delta \mathrm{V}_{E W}$ to correct this drift to is given [12].

$$
\Delta V_{E W}=3074.7 \frac{0.00171 \times 365}{3 \times 361}=1.77 \mathrm{~m} / \mathrm{s}
$$

Acceleration versus spacecraft longitude Longitude acceleration

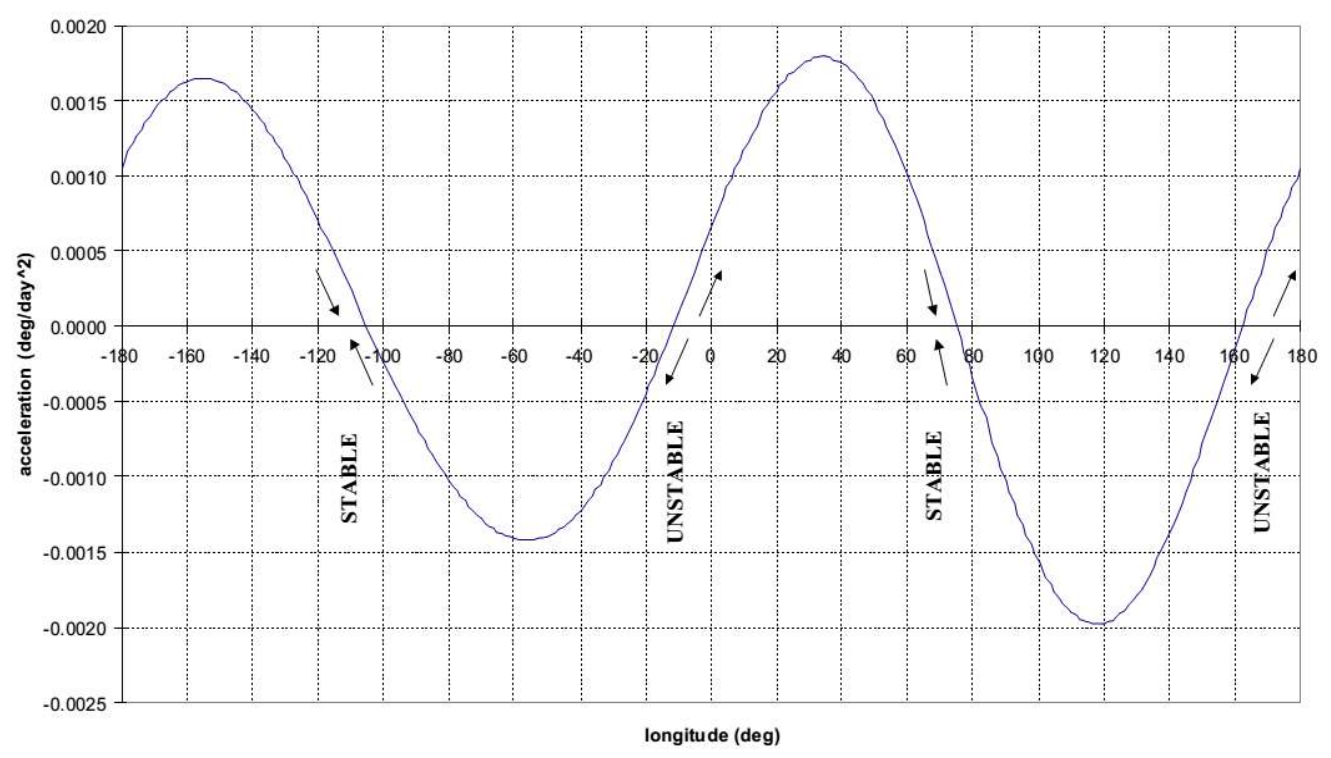

Figure 2. Longitude acceleration 
Natural evolution of yearly inclination changes between 0.75 and 0.95 degree (see Fig. 3). Average value of $0.85 \mathrm{deg} /$ year can be used for calculations. North-South maneuvers are performed to correct the inclination of the satellite. Yearly required $\Delta \mathrm{V}_{N S}$ to correct inclination is given as

$\Delta V_{N S}=3074.7 \times i=45.61 \mathrm{~m} / \mathrm{s}$

where " $i$ " is the inclination in radians [11].

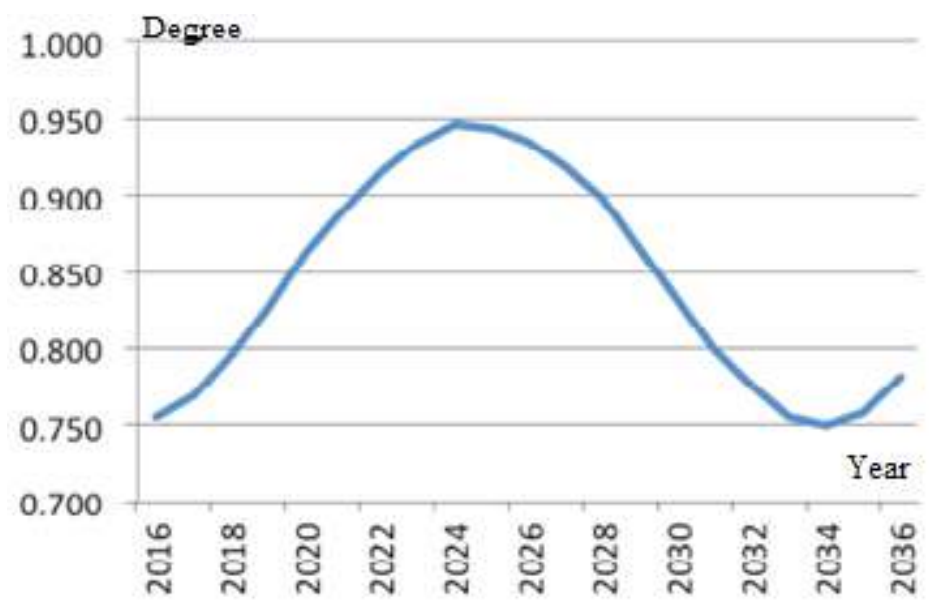

Figure 3. Yearly inclination change

ITU radio regulation requires deorbit of the geostationary satellite to an orbit with a perigee no less than $200 \mathrm{~km}$ above the geostationary altitude [13]. Calculation of perigee altitude $\Delta \mathrm{H}(\mathrm{km})$ is given in the ITU-R Recommendation

$\Delta H>235+1000 \cdot C_{r} \cdot \frac{A}{M}$

where

$\mathrm{Cr}$ is the reflectivity coefficient varying between 1 and 2,

A is the area $\left(\mathrm{m}^{2}\right)$,

$\mathrm{M}(\mathrm{kg})$ is the dry mass of the satellite.

The minimum perigee altitude can be calculated as $273 \mathrm{~km}$ for a typical geostationary satellite with $50 \mathrm{~m}^{2}$ solar panel area and $1500 \mathrm{~kg}$ dry mass. $350 \mathrm{~km}$ is a practical value used by satellite operators including margins. 
$\Delta \mathrm{V}$ for graveyard orbit is calculated using (1) with the assumption of Hohmann transfer to graveyard orbit. ITU recommends multiple maneuver strategy for orbit raising. First maneuver is performed to go from GEO orbit to a graveyard transfer orbit with apogee $42514 \mathrm{~km}$, perigee $42164 \mathrm{~km}$ and semi-major axis $42339 \mathrm{~km}$. Second maneuver is performed to circularize graveyard orbit with $42514 \mathrm{~km}$ radius.

$$
\begin{aligned}
& \Delta V_{1}=\sqrt{G M\left(\frac{2}{42164}-\frac{1}{42339}\right)}-V_{s} \\
& \Delta V_{2}=\sqrt{\frac{G M}{42514}}-\sqrt{G M\left(\frac{2}{42514}-\frac{1}{42339}\right)} \\
& \Delta V_{\text {deo }}=\Delta V_{1}+\Delta V_{2}=12.7 \mathrm{~m} / \mathrm{s}
\end{aligned}
$$

There will be dispersions caused by launcher orbit injection accuracy, apogee maneuver firing (AMF) and on-orbit maneuvers. Thruster pointing errors, North-South maneuvers cross-coupling effects, attitude control (wheel unloading) will require additional delta-V to correct dispersions. Dispersions are based on experience of the satellite manufacturer and can be assumed $2 \%$ of the total delta- $\mathrm{V}$ for propellant budget calculations.

Efficiency of the apogee motor and thrusters calculated statistically based on qualification test, finite thrust end usage experience. Efficiency of Apogee Boost Motor (ABM) and thrusters are assumed as 0.99 and 0.91 respectively.

\section{Propellant Budget Example}

Now propellant budget, can be calculated using orbit raising, East-West, North-South and deorbiting velocity increments. A lifetime of 15 years is targeted for an example calculation. Specific impulse of (Apogee Maneuver Firing) ABM for Apogee Maneuver Firing (AMF) is 320 and thrusters are assumed 292 second. Initial mass of the satellite is $4000 \mathrm{~kg}$. Required propellant is calculated using rocket equation and reduced from the satellite mass at each step. Velocity increment for East-West and North-South maneuvers are calculated by multiplying 15 year with (7) and (8). Dry mass is the mass of the satellite excluding the total propellant mass. Propellant Budget Example is shown in Table 2. 
Table 2. Propellant Budget Example-1

\begin{tabular}{|l|r|l|r|r|r|}
\hline Lifetime & 15 & years & & & \\
\hline Maneuver & DeltaV (m/s) & Isp (sec) & Efficiency & Delta M (kg) & Mass (kg) \\
\hline & & & & & 4000.0 \\
\hline AMF & 1495.7 & 320 & 0.99 & 1528.4 & 2471.6 \\
\hline $\mathrm{N} / \mathrm{S}$ & 684.15 & 292 & 0.91 & 570.7 & 1900.9 \\
\hline E/W & 26.55 & 292 & 0.91 & 19.3 & 1881.6 \\
\hline Deorbit & 12.7 & 292 & 0.91 & 9.1 & 1872.5 \\
\hline Dispersions & 44.4 & 292 & 0.91 & 31.6 & 1840.8 \\
\hline Propellant Mass & & & & 2159.2 & \\
\hline Dry Mass & & & & 1840.8 & \\
\hline
\end{tabular}

The methodology of propellant budget calculation presented in this study can be used in different scenarios. Dry mass of the satellite composed by system and payload equipment. Once the dry mass is estimated by evaluation of payload mission requirements such as number of the transponders and antennas, selection of propellant tanks is second major sizing decision at geostationary satellite design. Selection of propellant tanks is a result of required propellant mass for given lifetime. We can calculate required propellant mass and total mass of the satellite for $1250 \mathrm{~kg}$ dry mass and 15 years of lifetime as shown at Table 3.

Table 3. Propellant Budget Example-2

\begin{tabular}{|l|r|l|r|r|r|}
\hline Lifetime & 15 & years & & & \\
\hline Maneuver & DeltaV (m/s) & Isp (sec) & Efficiency & Delta M (kg) & Mass (kg) \\
\hline & & & & & 2716.1 \\
\hline AMF & 1495.7 & 320 & 0.99 & 1037.8 & 1678.3 \\
\hline $\mathrm{N} / \mathrm{S}$ & 684.15 & 292 & 0.91 & 387.5 & 1290.7 \\
\hline E/W & 26.55 & 292 & 0.91 & 13.1 & 1277.7 \\
\hline Deorbit & 12.7 & 292 & 0.91 & 6.2 & 1271.4 \\
\hline Dispersions & 44.4 & 292 & 0.91 & 21.5 & 1250.0 \\
\hline Propellant Mass & & & & 1466.1 & \\
\hline Dry Mass & & & & 1250.0 & \\
\hline
\end{tabular}

Another use of the propellant budget calculation is comparison of the different launchers for the same satellite. Since the GTO orbit parameters are different for launchers, requires delta-V for GEO orbit will differ, too. While Ariane-5 having 1496 m/s delta-V, Falcon-9 will require 1837 $\mathrm{m} / \mathrm{s}$ delta-V to reach GEO orbit. This difference will result 9 years of lifetime for the satellite if it is launched by Falcon-9.

Due to microgravity in space, remaining fuel in propellant tanks is calculated with bookkeeping or pressure, volume and temperature (PVT) methods. There will be some residual fuel in tubes and fuel vapor, which cannot be used for maneuver. Due to mixture ratio error, there will be remaining fuel or oxidizer in tanks while other tanks is empty. The remaining fuel is around $1 \%$ of total propellant and negligible for the propellant budget calculations at initial design phase. 


\section{Conclusion}

A guideline to calculate propellant budget of a geostationary satellite is presented in this paper. Presented approach is checked with various real satellites and has less than $1 \%$ accuracy for the propellant mass. Although the rocket equation is well known, this study provides a comprehensive guideline for the propellant calculation of geostationary satellites including orbit raising, on-orbit and deorbit maneuvers. Proposed methodology can be used practically in preliminary design phase of new geostationary satellite programs and comparison of the different launchers.

\section{References}

[1] Nelson R.A. Rocket Thrust Equation and Launch Vehicles, Via Satellite, June 1999.

[2] Curtis H, Orbital Mechanics for Engineering Students, 3rd Edition, Elsevier, 2014.

[3] Agrawal B.N., Design of Geosynchronous Spacecraft, Prentice-Hall, 1996.

[4] Griffinand M. D., French J.R., SpaceVehicle Design, AIAA Education series(ed. Joseph A. Schetz), 2004.

[5] Meyer R.X.,Elements of Space Technology for Aerospace Engineers, academic Press, 1999.

[6] Ley, K., Wittmann and W. Halmann (Editors), Handbook of Space Technology, Wiley, 2009.

[7] Campbell B.A and McCandless S.N., Introduction to Space science and Spacecraft Applications, Gulf Publishing Company 1996.

[8] Fortescue P, Swinerd G. and Stark J. (Editors), Spacecraft Systems Engineering, Wiley 2011.

[9] Maral G., Bousquet M., Satellite Communications Systems: Systems, Techniques and Technology, John Willey \& Sons, 2009.

[10] Ariane 5 User's Manual, Issue 5 Revision 2, October 2016.

[11] Falcon 9 Launch Vehicle Payload User's Guide, Rev 2, October 21, 2015.

[12] Soop E. M., Introduction to Geostationary Orbits, European Space Agency, 1983.

[13] Recommendation ITU-R S.1003-2 (12/2010), Enviromental protection of the geostsationary-satellite orbit. 